\title{
Differences in risk profiles and long-term outcomes in acute heart failure patients with preserved and reduced left ventricular ejection fraction in the Czech Republic: The AHEAD registry sub-analysis
}

\author{
Roman Mikliki, , Marie Miklikova ${ }^{\mathrm{c}, \mathrm{d}}$, Radim Spacek ${ }^{\mathrm{e}}$, Jindrich Spinar, ${ }^{\mathrm{c}, \mathrm{d}}$, Kamil Zeman ${ }^{\mathrm{e}}$, Klara Benesova ${ }^{\mathrm{f}}$, Marian Felsocic, \\ Lidka Pohludkova ${ }^{\mathrm{e}}$, Ladislav Dusek ${ }^{f}$, Jiri Jarkovsky', Petr Lokaj ${ }^{\mathrm{c}, \mathrm{d}}$, Ilona Parenicova ${ }^{\mathrm{g}}$, Jiri Parenica ${ }^{\mathrm{c}, \mathrm{d}}$
}

Background. The latest European heart failure guidelines define patients as those with reduced (HFrEF), mid-range, and preserved (HFpEF) left ventricular ejection fraction (LVEF; $<40 \%, 40 \%-49 \%$, and $\geq 50 \%$, respectively). We investigated the causes of rehospitalizations/deaths in our institution's heart failure patients and focused on differences in the clinical presentation, risk profile, and long-term outcomes between the HFrEF and HFpEF groups in a real-life scenario. Methods and Results. We followed 1274 patients discharged from heart failure hospitalization in 2 centres. The mean patient age was 75.9 years, and men and women were represented equally. During the minimal follow-up of 2 years, $57 \%$ of patients were hospitalised for any cause, $24.9 \%$ for decompensated heart failure, and $43.3 \%$ for any cardiovascular cause. A total of $36.1 \%$ of patients died, either with prior $(11.8 \%)$ or without prior (24.3\%) heart failure rehospitalization. Heart failure was also the most frequent cause of cardiovascular hospitalization, followed by gastrointestinal problems, infections, and tumours for noncardiovascular hospitalizations. Patients with HFrEF had different baseline characteristics and risk profiles, experienced more hospitalizations for acute heart failure (28.6\% vs $20.2 \%$, $P=0.012)$, and had higher cardiovascular mortality $(82.4 \%$ vs $63.5 \%, P<0.001)$ when compared with HFpEF patients. Overall mortality and rehospitalization rates were similar.

Conclusion. Within 2 years, half of the patients died and/or were hospitalised for acute decompensation of heart failure, and only one-third of the patients survived without any hospitalization. HFrEF and HFpEF patients were confirmed to be different entities with diverse characteristics, risk profiles, and cardiovascular event rates.

Key words: acute heart failure, left ventricular ejection fraction, rehospitalization, AHEAD, mortality

Received: June 14, 2020; Revised: August 30, 2020; Accepted: September 4, 2020; Available online: October 1, 2020 https://doi.org/10.5507/bp.2020.038

(c) 2021 The Authors; https://creativecommons.org/licenses/by/4.0/

${ }^{a}$ Cardiology, Podlesi Hospital Trinec, Czech Republic

'Internal Department, Military Hospital Brno, Czech Republic

'Department of Cardiology, University Hospital Brno, Czech Republic

${ }^{d}$ Medical Faculty, Masaryk University, Brno, Czech Republic

${ }^{e}$ Department of Internal Medicine, Hospital Frydek-Mistek, Frydek-Mistek, Czech Republic

fInstitute of Biostatistics and Analysis, Faculty of Medicine, Masaryk University, Brno, Czech Republic

${ }^{9}$ Centre of Cardiovascular and Transplant Surgery, Pekarska 53, Brno, Czech Republic

Corresponding author:Petr Lokaj, e-mail: alveus@seznam.cz

\section{INTRODUCTION}

The prognosis of patients hospitalised for acute heart failure (AHF) is unfavourable, with a hospitalization mortality rate of $4 \%$ to $12.7 \%$ (ref. ${ }^{1-4}$ ) and annual mortality of $17 \%$ to $32.3 \%$ (ref. $^{5}$ ) in an unselected population. The most frequent causes of death are sudden cardiac death and terminal progression of heart failure.

Heart failure is a global problem with a rapidly increasing number of diagnosed patients; in 2012, the estimated number of patients worldwide was 26 million. Hospitalization for heart failure is the major cause of hospitalizations in the United States and Europe. There are more than 1 million AHF hospitalizations per year, accounting for $1 \%$ to $2 \%$ of all hospitalizations $s^{6-13}$ and consuming as much as $7.7 \%$ of all annual in-hospital expenses and $2.7 \%$ of total healthcare expenditure ${ }^{14}$.
Systolic function of the left ventricle plays an important role in a patient's prognosis. New guidelines from the European Society of Cardiology for the Diagnosis and Treatment of Heart Failure were published in 2016 $\left(\right.$ ref. $\left.^{2}\right)$. Compared with previous guidelines, a new group of patients with mid-range left ventricular ejection fraction (LVEF; from 40 to $49 \%$ ) was defined as a transition group between those with preserved (HFpEF; LVEF $250 \%$ ) and reduced systolic function (HFrEF; LVEF <40\%).

To our knowledge, no article has been published to date comparing long-term outcomes of patients with preserved and reduced LVEF according to the new guidelines who were discharged in a stable condition from an AHF hospitalization. The primary objective of our study was to describe the combined incidence of rehospitalizations for acute decompensation of heart failure and overall mortality during a 2-year follow-up. The secondary ob- 


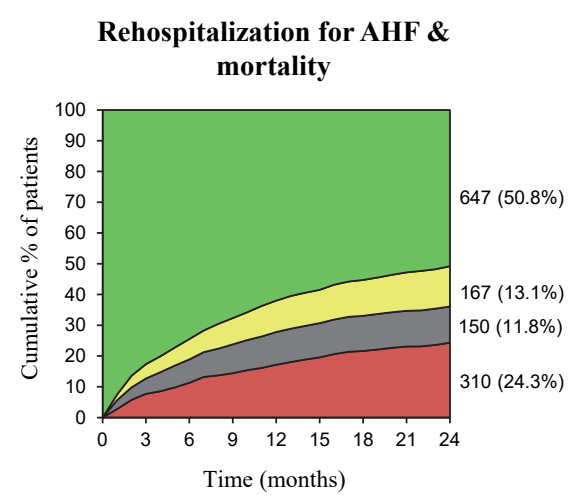

No death, no hospitalization

Rehospitalization without death

Rehospitalization with death afterwards

Death without prior rehospitalization
Rehospitalization for CV cause

(AHF, ACS, other CV causes)

\& mortality

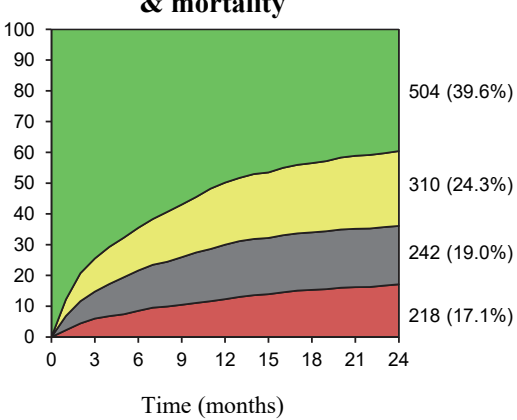

Rehospitalization for any

cause \& mortality

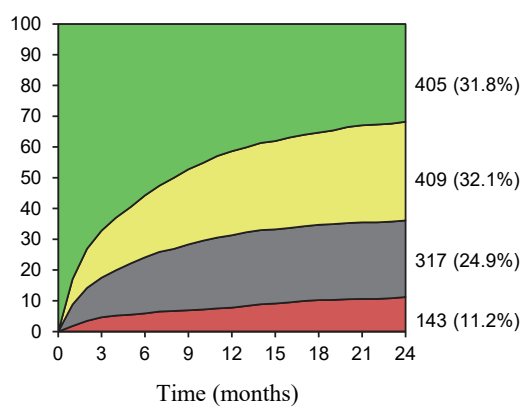

Fig. 1. Rehospitalization \& mortality within 2 years after discharge from index acute heart failure hospitalization $(n=1274)$. ACS - acute coronary syndrome; AHF - acute heart failure; CV - cardiovascular

jectives were the number of cardiovascular and all-cause hospitalizations, mortality rates, and causes of death. Furthermore, we focused in detail on the comparison between characteristics, risk profiles, and outcomes of patients with HFrEF and HFpEF.

\section{METHODS}

Our study was a retrospective analysis of a set of 1,274 consecutive patients enrolled in the AHEAD registry (Czech Acute Heart Failure Database) who were discharged to home in a stable condition from a hospitalization for AHF. These patients were considered as chronic heart failure patients and the hospitalization hereinafter referred to as the index hospitalization. The Acute Heart Failure Database included patients enrolled from 20062012 in 15 participating centres; the methodology has been described in detail elsewhere ${ }^{15,16}$. Briefly, the exclusion criteria were advanced stage of malignancy (life expectancy less than 2 years) and refusal to participate in the registry. Nearly all consecutive patients with a final diagnosis of AHF according to the European Acute Heart Failure Guidelines issued in 2005 (ref. ${ }^{10}$ ) were enrolled during the index hospitalization. In this subanalysis, we evaluated consecutive patients from two centres only. These centres had collected more extensive follow-up data of enrolled patients than the other sites. As one centre is a major district hospital with a cath lab (Brno University Hospital) and the other is a regional referring hospital without a cath lab (Frydek-Mistek Hospital), our study cohort can be considered as a representative sample of the entire population.

The minimal follow-up was 2 years and was based on regular outpatient check-ups, evaluation of hospitalization records, and phone consultations with a patient or his or her relative. Causes of death were identified from the Institute of Health Information and Statistics of the
Czech Republic. We assume to have captured more than $95 \%$ of all hospitalization events.

The study was approved by the Ethics Committee of Brno University Hospital and was conducted in accordance with the provisions of the Declaration of Helsinki. All patients signed an informed consent to participate in the registry.

Subsequent hospitalizations were divided into 4 categories: (1) AHF hospitalization, (2) acute coronary syndrome (ACS) hospitalization, (3) hospitalization for other cardiovascular cause, and (4) noncardiovascular hospitalization.

The patients were also divided into 4 groups by their outcome: (1) no death, no hospitalization; (2) rehospitalization without death; (3) rehospitalization with death afterwards; (4) death without prior rehospitalization.

To provide a detailed analysis of patient outcomes based on LVEF, the patients were divided into 3 groups: (1) HFrEF (LVEF <40\%), (2) mid-range (LVEF 40\%$49 \%$ ), and (3) HFpEF (LVEF > 50\%).

\section{Statistical analysis}

Standard descriptive statistics were used to analyse the data. Categorical variables were described by absolute and relative frequencies and continuous variables by median and 5th-95th percentile range or by average with standard deviation. A statistical significance of differences between patient groups was tested by the KruskalWallis test and post hoc tests for continuous variables and by chi-square test or Fisher's exact test for categorical variables. Survival (time-to-event) analysis was used to evaluate the likelihood of hospitalizations and deaths at certain time points; Kaplan-Meyer curves were used for graphical visualisation. Binary logistic regression models and subsequently multivariate logistic regression models using backward stepwise algorithm for selection of independent predictors were used to calculate the odds of 2-year outcomes for different variables stratified by the 
Table 1. Basic characteristics of 1274 consecutive patients discharged from hospitalization for acute heart failure in a stabilized condition. A total of 1166 (91.5\%) patients had echocardiography performed during index hospitalization.

\begin{tabular}{|c|c|c|c|c|c|}
\hline & $\begin{array}{l}\text { Patients in total } \\
(\mathrm{n}=1274)\end{array}$ & $\begin{array}{c}\text { LVEF < 40\% } \\
(n=482)\end{array}$ & $\begin{array}{l}\text { LVEF 40-49\% } \\
\quad(n=244)\end{array}$ & $\begin{array}{c}\text { LVEF } \geq 50 \% \\
(n=440)\end{array}$ & $P$ \\
\hline Gender - male & $647(50.8 \%)$ & $311(64.5 \%)$ & $137(56.1 \%)$ & $157(35.7 \%)$ & $<0.001$ \\
\hline Age (years) & $75.9(54.3 ; 88.4)$ & $73.8(50.8 ; 87.2)$ & $75.0(54.8 ; 88.4)$ & $76.9(55.3 ; 87.7$ & $<0.001$ \\
\hline BMI $\left(\mathrm{kg} / \mathrm{m}^{2}\right)$ & $\begin{array}{r}\mathrm{n}=1005 ; \\
27.9(21.1 ; 39.8)\end{array}$ & $\begin{array}{r}\mathrm{n}=389 \\
27.7(21.1 ; 38.9)\end{array}$ & $\begin{array}{r}\mathrm{n}=203 \\
27.9(21.3 ; 40.1)\end{array}$ & $\begin{array}{r}\mathrm{n}=347 ; \\
28.4(21.0 ; 41.1)\end{array}$ & 0.246 \\
\hline Systolic pressure $(\mathrm{mmHg})$ & $\begin{array}{r}\mathrm{n}=1259 \\
140.0(95.0 \\
200.0)\end{array}$ & $\begin{array}{r}\mathrm{n}=475 ; \\
140.0(90.0 ; 190.0)\end{array}$ & $\begin{array}{r}\mathrm{n}=244 \\
150.0(100.0 ; 220.0)\end{array}$ & $\begin{array}{r}\mathrm{n}=434 ; \\
145.0(95.0 ; 210.0)\end{array}$ & $<0.001$ \\
\hline Diastolic pressure $(\mathrm{mmHg})$ & $\begin{array}{r}\mathrm{n}=1257 ; \\
80.0(60.0 ; 110.0)\end{array}$ & $\begin{array}{r}\mathrm{n}=475 \\
80.0(60.0 ; 110.0)\end{array}$ & $\begin{array}{r}\mathrm{n}=244 \\
80.0(60.0 ; 120.0)\end{array}$ & $\begin{array}{r}\mathrm{n}=433 \\
80.0(60.0 ; 115.0)\end{array}$ & 0.309 \\
\hline Heart rate (per min) & $\begin{array}{r}\mathrm{n}=1266 \\
95.0(60.0 ; 150.0)\end{array}$ & $\begin{array}{r}\mathrm{n}=478 \\
100.0(60.0 ; 160.0)\end{array}$ & $\begin{array}{r}\mathrm{n}=242 \\
90.0(57.0 ; 135.0)\end{array}$ & $\begin{array}{r}\mathrm{n}=438 \\
90.0(54.0 ; 150.0)\end{array}$ & $<0.001$ \\
\hline \multicolumn{6}{|l|}{ NYHA class dyspnea } \\
\hline 1 & $6.2 \%)$ & $73(15.2 \%)$ & $50(20.6 \%)$ & $76(17.4 \%)$ & \multirow{3}{*}{0.006} \\
\hline 2 & $880(69.4 \%)$ & $317(65.9 \%)$ & $161(66.3 \%)$ & $313(71.6 \%)$ & \\
\hline 3 & $183(14.4 \%)$ & $91(18.9 \%)$ & $32(13.2 \%)$ & $48(11.0 \%)$ & \\
\hline Hypertension & $1001(79.1 \%)$ & $370(76.8 \%)$ & $189(77.5 \%)$ & $357(81.1 \%)$ & 0.243 \\
\hline Diabetes mellitus & $551(43.5 \%)$ & $203(42.1 \%)$ & $101(41.4 \%)$ & $195(44.3 \%)$ & 0.703 \\
\hline Prior myocardial infarction & $395(31.2 \%)$ & $191(39.6 \%)$ & $88(36.1 \%)$ & $85(19.3 \%)$ & $<0.001$ \\
\hline Prior PCI/CABG & $208(16.4 \%)$ & $97(20.1 \%)$ & $47(19.3 \%)$ & $43(9.8 \%)$ & $<0.001$ \\
\hline Stroke/TIA & $323(25.5 \%)$ & $107(22.2 \%)$ & $57(23.4 \%)$ & $119(27.0 \%)$ & 0.215 \\
\hline Peripheral artery disease & $302(23.8 \%)$ & $125(25.9 \%)$ & $48(19.7 \%)$ & $95(21.6 \%)$ & 0.113 \\
\hline COPD & $309(24.4 \%)$ & $117(24.3 \%)$ & $62(25.4 \%)$ & $107(24.3 \%)$ & 0.937 \\
\hline Atrial fibrillation & $443(34.9 \%)$ & $160(33.2 \%)$ & $68(27.9 \%)$ & $173(39.3 \%)$ & 0.008 \\
\hline (pg/ml; baseline) & $\begin{array}{r}(\mathrm{n}=234) \\
788 \\
(86 ; 4559)\end{array}$ & $\begin{array}{r}\mathrm{n}=96 ; \\
1187.9 \\
(147.9 ; 5000.0)\end{array}$ & $\begin{array}{r}\mathrm{n}=37 ; \\
576.5 \\
(180.0 ; 2908.2)\end{array}$ & $\begin{array}{r}\mathrm{n}=76 ; \\
536.8 \\
(44.7 ; 3907.0)\end{array}$ & $<0.001$ \\
\hline $\mathrm{eGFR}\left(\mathrm{mL} / \mathrm{min} / 1.73 \mathrm{~m}^{2}\right)$ & $52.7(21.2 ; 96.6)$ & $52.7(22.8 ; 95.3)$ & $56.2(23.9 ; 101.3)$ & $51.6(18.0 ; 89.4)$ & 0.007 \\
\hline LVEF (\%) & $42(20 ; 65)$ & - & - & - & - \\
\hline Acute heart failure de-novo & $621(48.7 \%)$ & $196(40.7 \%)$ & $124(50.8 \%)$ & $268(60.9 \%)$ & $<0.001$ \\
\hline \multicolumn{6}{|l|}{ Acute heart failure etiology } \\
\hline Acute coronary syndrome & $285(22.4 \%)$ & $111(23.0 \%)$ & $78(32.0 \%)$ & $82(18.6 \%)$ & \multirow[t]{6}{*}{$<0.001$} \\
\hline Ischemic heart di & $348(27.3 \%)$ & $188(39.0 \%)$ & $73(29.9 \%)$ & $40(9.1 \%)$ & \\
\hline Valve defects & $178(14.0 \%)$ & $43(8.9 \%)$ & $34(13.9 \%)$ & $91(20.7 \%)$ & \\
\hline Arrhythmia & $166(13.0 \%)$ & $47(9.8 \%)$ & $20(8.2 \%)$ & $83(18.9 \%)$ & \\
\hline Hypertension & $79(6.2 \%)$ & $6(1.2 \%)$ & $19(7.8 \%)$ & $46(10.5 \%)$ & \\
\hline Other & $218(17.1 \%)$ & $87(18.0 \%)$ & $20(8.2 \%)$ & $98(22.3 \%)$ & \\
\hline \multicolumn{6}{|l|}{ Hospitalization procedures } \\
\hline Pacemaker & $39(3.1 \%)$ & $15(3.1 \%)$ & $9(3.7 \%)$ & $12(2.7 \%)$ & 0.784 \\
\hline PCI & $143(11.2 \%)$ & $57(11.8 \%)$ & $41(16.8 \%)$ & $44(10.0 \%)$ & 0.032 \\
\hline $\mathrm{CABC}$ & $38(3.0 \%)$ & $19(3.9 \%)$ & $15(6.1 \%)$ & $4(0.9 \%)$ & $<0.001$ \\
\hline ICD & $24(1.9 \%)$ & $7(1.5 \%)$ & $1(0.4 \%)$ & $3(0.7 \%)$ & 0.301 \\
\hline CRT & $11(0.9 \%)$ & $9(1.9 \%)$ & $0(0.0 \%)$ & $0(0.0 \%)$ & 0.002 \\
\hline \multicolumn{6}{|l|}{ Medication at discharge } \\
\hline Antiaggregation & $905(71.3 \%)$ & $342(71.0 \%)$ & $196(80.3 \%)$ & $285(64.8 \%)$ & $<0.001$ \\
\hline Anticoagulation & $312(24.6 \%)$ & $122(25.3 \%)$ & $51(20.9 \%)$ & $124(28.2 \%)$ & 0.111 \\
\hline ACE-inhibitors/sartans & $1064(83.8 \%)$ & $416(86.3 \%)$ & $207(84.8 \%)$ & $355(80.7 \%)$ & 0.061 \\
\hline Beta-blockers & $954(75.2 \%)$ & $394(81.7 \%)$ & $195(79.9 \%)$ & $297(67.5 \%)$ & $<0.001$ \\
\hline Calcium channel blockers & $286(22.5 \%)$ & $61(12.7 \%)$ & $53(21.7 \%)$ & $146(33.2 \%)$ & $<0.001$ \\
\hline Diuretics & $1127(88.8 \%)$ & $452(93.8 \%)$ & $210(86.1 \%)$ & $368(83.6 \%)$ & $<0.001$ \\
\hline Verospirone & $578(45.5 \%)$ & $318(66.0 \%)$ & $98(40.2 \%)$ & $120(27.3 \%)$ & $<0.001$ \\
\hline Digoxin & $310(24.4 \%)$ & $143(29.7 \%)$ & $51(20.9 \%)$ & $84(19.1 \%)$ & $<0.001$ \\
\hline
\end{tabular}

Categorical variables are described by absolute (relative) frequencies. Continuous variables are described by n, median (5th-95th percentile). BNP - B-type natriuretic peptide; CABG - coronary bypass graft; COPD - chronic obstructive pulmonary disease; CRT - resynchronization therapy; eGFR - estimated glomerular filtration rate by Modification of Diet in Renal Disease study equation; ICD - implantable cardioverter defibrillator; LVEF - left ventricular ejection fraction; PCI - percutaneous coronary intervention; PM - pacemaker; TIA - transient ischemic attack

* Reported \% are related to total number of hospitalizations in the 1 st year $(n=1193)$, in the $2^{\text {nd }}$ year $(n=318)$ and the sum of both years $(n=1511)$. The column totals can exceed $100 \%$ as there could have been 2 major causes for one hospitalization. 
Table 2. Number of hospitalizations during the first two years following discharge from index hospitalization $(\mathrm{n}=1274)$.

\begin{tabular}{lccccc}
\hline $\begin{array}{l}\text { Number } \\
\text { of hospitalizations }\end{array}$ & AHF & ACS & Cause of hospitalization \\
\hline $0 . \mathrm{n}(\%)$ & $957(75.1 \%)$ & $1209(94.9 \%)$ & $921(72.3 \%)$ & $722(56.7 \%)$ & $548(43.0 \%)$ \\
$1 . \mathrm{n}(\%)$ & $225(17.7 \%)$ & $50(3.9 \%)$ & $233(18.3 \%)$ & $349(27.4 \%)$ & $362(28.4 \%)$ \\
$2 . \mathrm{n}(\%)$ & $57(4.5 \%)$ & $12(0.9 \%)$ & $75(5.9 \%)$ & $112(8.8 \%)$ & $182(14.3 \%)$ \\
$3 . \mathrm{n}(\%)$ & $22(1.7 \%)$ & $3(0.2 \%)$ & $19(1.5 \%)$ & $53(4.2 \%)$ & $89(7.0 \%)$ \\
> 3. n (\%) & $13(1.0 \%)$ & $0(0.0 \%)$ & $26(2.0 \%)$ & $38(3.0 \%)$ & $93(7.3 \%)$ \\
\hline Average (SD) & $0.4(0.8)$ & $0.1(0.3)$ & $0.5(1.0)$ & $0.7(1.1)$ & $1.2(1.6)$ \\
Median (min.-max.) & $0(0 ; 11)$ & $0(0 ; 3)$ & $0(0 ; 14)$ & $0(0 ; 11)$ & $1(0 ; 15)$ \\
\hline
\end{tabular}

ACS - acute coronary syndrome; AHF - acute heart failure; CV - cardiovascular; SD - standard deviation

Table 3. The causes for hospitalizations during the first and the second year of follow-up and cumulative summary of all events during the follow-up period of 2 years after index hospital discharge.

\begin{tabular}{|c|c|c|c|c|c|c|}
\hline \multirow[t]{2}{*}{$\begin{array}{l}\text { Cause for hospitalization } \\
n=1274\end{array}$} & \multicolumn{2}{|c|}{$\begin{array}{l}\text { Number of hospitalizations } \\
\text { in } 1^{\text {st }} \text { year }\end{array}$} & \multicolumn{2}{|c|}{$\begin{array}{l}\text { Number of hospitalizations } \\
\text { in } 2^{\text {nd }} \text { year }\end{array}$} & \multicolumn{2}{|c|}{$\begin{array}{l}\text { Cumulative number } \\
\text { of all hospitalizations }\end{array}$} \\
\hline & Total $^{*}$ & $\begin{array}{c}\text { Per } 1000 \\
\text { patients }\end{array}$ & Total* & $\begin{array}{l}\text { Per } 1000 \\
\text { patients }\end{array}$ & Total* & $\begin{array}{r}\text { Per } 1000 \\
\text { patients }\end{array}$ \\
\hline CV AHF & $364(30.5 \%)$ & 286 & $108(34.0 \%)$ & 85 & $472(31.2 \%)$ & 370 \\
\hline ACS & $72(6.0 \%)$ & 57 & $21(6.6 \%)$ & 16 & $93(6.2 \%)$ & 73 \\
\hline \multicolumn{7}{|l|}{ Other } \\
\hline Arrhythmia & $124(10.4 \%)$ & 97 & $33(10.4 \%)$ & 26 & $157(10.4 \%)$ & 123 \\
\hline CVD & $58(4.9 \%)$ & 46 & $20(6.3 \%)$ & 16 & $78(5.2 \%)$ & 61 \\
\hline PAD & $41(3.4 \%)$ & 32 & $9(2.8 \%)$ & 7 & $50(3.3 \%)$ & 39 \\
\hline Hypertension & $20(1.7 \%)$ & 16 & $7(2.2 \%)$ & 5 & $27(1.8 \%)$ & 21 \\
\hline Valve disease & $20(1.7 \%)$ & 16 & $1(0.3 \%)$ & 1 & $21(1.4 \%)$ & 16 \\
\hline CAD & $16(1.3 \%)$ & 13 & $1(0.3 \%)$ & 1 & $17(1.1 \%)$ & 13 \\
\hline PM/ICD implantation & $14(1.2 \%)$ & 11 & $9(2.8 \%)$ & 7 & $23(1.5 \%)$ & 18 \\
\hline Pulmonary embolism & $13(1.1 \%)$ & 10 & $1(0.3 \%)$ & 1 & $14(0.9 \%)$ & 11 \\
\hline Deep venous thrombosis & $9(0.8 \%)$ & 7 & $0(0.0 \%)$ & 0 & $9(0.6 \%)$ & 7 \\
\hline Systemic embolism & $4(0.3 \%)$ & 3 & $1(0.3 \%)$ & 1 & $5(0.3 \%)$ & 4 \\
\hline PCI & $3(0.3 \%)$ & 2 & $3(0.9 \%)$ & 2 & $6(0.4 \%)$ & 5 \\
\hline Other & $3(0.3 \%)$ & 2 & $4(1.3 \%)$ & 3 & $7(0.5 \%)$ & 5 \\
\hline \multicolumn{7}{|l|}{ Non CV } \\
\hline GIT disease & $90(7.5 \%)$ & 71 & $9(2.8 \%)$ & 7 & $99(6.6 \%)$ & 78 \\
\hline Respiratory infection & $57(4.8 \%)$ & 45 & $17(5.3 \%)$ & 13 & $74(4.9 \%)$ & 58 \\
\hline Tumor & $55(4.6 \%)$ & 43 & $19(6.0 \%)$ & 15 & $74(4.9 \%)$ & 58 \\
\hline Diabetes mellitus & $40(3.4 \%)$ & 31 & $16(5.0 \%)$ & 13 & $56(3.7 \%)$ & 44 \\
\hline Bleeding & $38(3.2 \%)$ & 30 & $6(1.9 \%)$ & 5 & $44(2.9 \%)$ & 35 \\
\hline Renal failure/disease & $30(2.5 \%)$ & 24 & $9(2.8 \%)$ & 7 & $39(2.6 \%)$ & 31 \\
\hline Non-respiratory infection & $27(2.3 \%)$ & 21 & $8(2.5 \%)$ & 6 & $35(2.3 \%)$ & 27 \\
\hline Dehydratation & $19(1.6 \%)$ & 15 & $2(0.6 \%)$ & 2 & $21(1.4 \%)$ & 16 \\
\hline COPD & $19(1.6 \%)$ & 15 & $6(1.9 \%)$ & 5 & $25(1.7 \%)$ & 20 \\
\hline Urinary infection & $15(1.3 \%)$ & 12 & $7(2.2 \%)$ & 5 & $22(1.5 \%)$ & 17 \\
\hline Anemia & $14(1.2 \%)$ & 11 & $1(0.3 \%)$ & 1 & $15(1.0 \%)$ & 12 \\
\hline Syncope & $14(1.2 \%)$ & 11 & $6(1.9 \%)$ & 5 & $20(1.3 \%)$ & 16 \\
\hline Thoracic syndrome & $11(0.9 \%)$ & 9 & $1(0.3 \%)$ & 1 & $12(0.8 \%)$ & 9 \\
\hline Musculoskeletal pain & $10(0.8 \%)$ & 8 & $2(0.6 \%)$ & 2 & $12(0.8 \%)$ & 9 \\
\hline Neurological disease & $10(0.8 \%)$ & 8 & $1(0.3 \%)$ & 1 & $11(0.7 \%)$ & 9 \\
\hline Fracture & $7(0.6 \%)$ & 5 & $0(0.0 \%)$ & 0 & $7(0.5 \%)$ & 5 \\
\hline Collapse & $5(0.4 \%)$ & 4 & $0(0.0 \%)$ & 0 & $5(0.3 \%)$ & 4 \\
\hline Diagnostic hospitalization & $0(0.0 \%)$ & 0 & $1(0.3 \%)$ & 1 & $1(0.1 \%)$ & 1 \\
\hline Other & $56(4.7 \%)$ & 44 & $23(7.2 \%)$ & 18 & $79(6.0 \%)$ & 62 \\
\hline Total & $1193(100.0 \%)$ & 936 & $318(100.0 \%)$ & 250 & $1511(100.0 \%)$ & 1186 \\
\hline
\end{tabular}

ACS - acute coronary syndrome; AHF - acute heart failure; CAD - chronic coronary artery disease; COPD - chronic obstructive pulmonary disease; CV - cardiovascular; CVD - cerebrovascular disease; GIT - gastrointestinal tract; PCI - percutaneous coronary intervention; PM/ICD - pacemaker / implantable cardioverter-defibrillator.

* Reported \% are related to total number of hospitalizations in the 1 st year $(n=1193)$, in the 2 nd year $(n=318)$ and the sum of both years $(n=$ 1511). The column totals can exceed $100 \%$ as there could have been 2 major causes for one hospitalization. 
Table 4. Echocardiographical findings - diastolic function and valvular defects by LVEF groups.

Only moderate-severe defects are reported.

\begin{tabular}{|c|c|c|c|c|}
\hline & $\begin{array}{c}\text { LVEF < 40\% } \\
(\mathrm{n}=482)\end{array}$ & $\begin{array}{c}\text { LVEF 40-49\% } \\
\quad(n=244) \\
\end{array}$ & $\begin{array}{c}\text { LVEF } \geq 50 \% \\
(n=440)\end{array}$ & $P$ \\
\hline \multicolumn{5}{|l|}{ Diastolic function } \\
\hline Normal & $29(6.0 \%)$ & $25(10.2 \%)$ & $29(6.6 \%)$ & \multirow{5}{*}{$<0.001$} \\
\hline Relaxation failure & $80(16.6 \%)$ & $76(31.1 \%)$ & $190(43.2 \%)$ & \\
\hline Pseudonormalisation & $29(6.0 \%)$ & $11(4.5 \%)$ & $19(4.3 \%)$ & \\
\hline Restriction & $69(14.3 \%)$ & $10(4.1 \%)$ & $18(4.1 \%)$ & \\
\hline Unknown & $275(57.1 \%)$ & $122(50.0 \%)$ & $184(41.8 \%)$ & \\
\hline \multicolumn{5}{|l|}{ Valvular defects } \\
\hline Mitral regurgitation & $279(57.9 \%)$ & $95(38.9 \%)$ & $142(32.3 \%)$ & $<0.001$ \\
\hline Mitral stenosis & $4(0.8 \%)$ & $3(1.2 \%)$ & $14(3.2 \%)$ & 0.031 \\
\hline Aortic stenosis & $41(8.5 \%)$ & $18(7.4 \%)$ & $39(8.9 \%)$ & 0.831 \\
\hline Aortic regurgitation & $19(3.9 \%)$ & $12(4.9 \%)$ & $35(8.0 \%)$ & 0.030 \\
\hline Tricuspid regurgitation & $204(42.3 \%)$ & $62(25.4 \%)$ & $142(32.3 \%)$ & $<0.001$ \\
\hline Other valvular disease & $5(1.0 \%)$ & $6(2.5 \%)$ & $8(1.8 \%)$ & 0.309 \\
\hline
\end{tabular}

LVEF - left ventricular ejection fraction

$P$-value of Fisher Exact Test is reported.

Table 5. Rehospitalization \& mortality within 2 years after discharge from index acute heart failure hospitalization, stratified by LVEF.

\begin{tabular}{|c|c|c|c|c|c|}
\hline 2-year outcome & $\begin{array}{c}\text { LVEF }<40 \% \\
(n=482)\end{array}$ & $\begin{array}{l}\text { LVEF } 40-49 \% \\
\quad(\mathrm{n}=244)\end{array}$ & $\begin{array}{c}\text { LVEF } \geq 50 \% \\
(n=440)\end{array}$ & $P$ & \\
\hline \multicolumn{6}{|l|}{ Rehospitalizations } \\
\hline Rehospitalization for any cause & $269(55.8 \%)$ & $132(54.1 \%)$ & $270(61.4 \%)$ & 0.110 & \\
\hline Rehospitalization for CV cause & $220(45.6 \%)$ & $99(40.6 \%)$ & $190(43.2 \%)$ & 0.418 & \\
\hline Rehospitalization for AHF & $138(28.6 \%)$ & $62(25.4 \%)$ & $89(20.2 \%)$ & 0.012 & $\mathrm{a} / \mathrm{ab} / \mathrm{b}$ \\
\hline Rehospitalizations and mortality & $182(37.8 \%)$ & $74(30.3 \%)$ & $148(33.6 \%)$ & 0.120 & \\
\hline Rehospitalization for any cause or mortality & $328(68.0 \%)$ & $151(61.9 \%)$ & $311(70.7 \%)$ & 0.063 & \\
\hline Rehospitalization for CV cause or mortality & $301(62.4 \%)$ & $127(52.0 \%)$ & $267(60.7 \%)$ & 0.023 & $\mathrm{a} / \mathrm{b} / \mathrm{a}$ \\
\hline Rehospitalization for AHF or mortality & $256(53.1 \%)$ & $109(44.7 \%)$ & $195(44.3 \%)$ & 0.014 & $a / b / b$ \\
\hline
\end{tabular}

LVEF - left ventricular ejection fraction, CV - cardiovascular, AHF - acute heart failure

$P$-value of Fisher Exact Test is reported; post hoc - same letters denote groups which do not differ statistically from each other.

patients' LVEF. The level of significance was set at $\alpha=$ 0.05 for all analyses.

\section{RESULTS}

\section{Entire cohort (1274 patients with AHF)}

Baseline characteristics, course of hospitalization, medication

The patients' baseline characteristics are shown in Table 1 (left column). The mean patient age was 75.9 years, and half of the patients were men. The most frequent comorbidities included hypertension (79.1\%), diabetes mellitus (43.5\%), and prior myocardial infarction (31.2\%). A quarter of the patients had previous stroke, chronic obstructive pulmonary disease, and peripheral ischemic disease; one-third had any form of atrial fibrillation. ACS (22.4\%) and chronic coronary artery disease (27.3\%) were the most common causes of AHF hospitalizations, followed by valvular defects (14\%) and arrhythmias (13\%). At discharge from the index hospitalization, most patients had their heart failure medication well established.

Outcome

During the course of the 2-year follow-up, the primary combined endpoint (AHF rehospitalization/all-cause death) occurred in $49.2 \%$ of the patients. Fifty-seven percent of the patients were hospitalised for any cause, $24.9 \%$ for acute decompensation of heart failure and $43.3 \%$ for any cardiovascular cause; only $31.8 \%$ of the patients survived without any rehospitalization. The overall mortality rate was $36.1 \%$ ( $11.8 \%$ with prior and $24.3 \%$ without prior heart failure rehospitalization; Fig 1).

Table 2 clearly shows that some patients experienced multiple rehospitalizations. More than 3 rehospitalizations for heart failure decompensation were recorded in $1 \%$ of the patients, 3 rehospitalizations in $1.7 \%$, and 2 rehospitalizations in $4.5 \%$. The mean number of rehospitalizations for acute decompensation of heart failure was $0.4 \pm 0.8$ per patient, for a cardiovascular cause $0.5 \pm 1.0$, and for any cause $1.2 \pm 1.6$ per patient over the period of 2 years. 
Table 6. Causes of death by LVEF, as reported in hospitalization records or in the Institute of Health Information and Statistics of the Czech Republic.

\begin{tabular}{|c|c|c|c|c|}
\hline & $\begin{array}{l}\mathrm{EF}<40 \% \\
(\mathrm{n}=482)\end{array}$ & $\begin{array}{l}\text { EF } 40-49 \% \\
(n=244)\end{array}$ & $\begin{array}{l}E F \geq 50 \% \\
(n=440)\end{array}$ & $P$ \\
\hline No. of deaths within the first two years of follow-up & $182(37.8 \%)$ & $74(30.3 \%)$ & $148(33.6 \%)$ & \\
\hline \multicolumn{5}{|l|}{$\%$ and $P$-values related to patients who died: } \\
\hline Diseases of the circulatory system (I00-I99) & $150(82.4 \%)$ & $54(73.0 \%)$ & $94(63.5 \%)$ & 0.001 \\
\hline Neoplasms (C00-D49) & $12(6.6 \%)$ & $7(9.5 \%)$ & $21(14.2 \%)$ & 0.073 \\
\hline Diseases of the respiratory system (J00-J99) & $6(3.3 \%)$ & $6(8.1 \%)$ & $8(5.4 \%)$ & 0.252 \\
\hline Endocrine, nutritional and metabolic diseases (E00-E89) & $6(3.3 \%)$ & $1(1.4 \%)$ & $9(6.1 \%)$ & 0.237 \\
\hline Diseases of the digestive system (K00-K95) & $2(2.2 \%)$ & $2(4.1 \%)$ & $5(4.1 \%)$ & 0.365 \\
\hline $\begin{array}{l}\text { Injury, poisoning and certain other consequences of external causes } \\
\text { (S00-T88) }\end{array}$ & $2(1.1 \%)$ & $1(2.7 \%)$ & $5(3.4 \%)$ & 0.370 \\
\hline Other cause of death & $4(1.1 \%)$ & $3(1.4 \%)$ & $6(3.4 \%)$ & 0.571 \\
\hline \multicolumn{5}{|l|}{$\%$ and $P$-values related to all patients: } \\
\hline Diseases of the circulatory system (I00-I99) & $150(31.1 \%)$ & $54(22.1 \%)$ & $94(21.4 \%)$ & 0.001 \\
\hline Neoplasms (C00-D49) & $12(2.5 \%)$ & $7(2.9 \%)$ & $21(4.8 \%)$ & 0.150 \\
\hline Diseases of the respiratory system (J00-J99) & $6(1.2 \%)$ & $6(2.5 \%)$ & $8(1.8 \%)$ & 0.434 \\
\hline Endocrine, nutritional and metabolic diseases (E00-E89) & $6(1.2 \%)$ & $1(0.4 \%)$ & $9(2.0 \%)$ & 0.209 \\
\hline Diseases of the digestive system (K00-K95) & $2(0.8 \%)$ & $2(1.2 \%)$ & $5(1.4 \%)$ & 0.465 \\
\hline $\begin{array}{l}\text { Injury, poisoning and certain other consequences of external } \\
\text { causes (S00-T88) }\end{array}$ & $2(0.4 \%)$ & $1(0.8 \%)$ & $5(1.1 \%)$ & 0.429 \\
\hline Other cause of death & $4(0.4 \%)$ & $3(0.4 \%)$ & $6(1.1 \%)$ & 0.718 \\
\hline
\end{tabular}

LVEF - left ventricular ejection fraction

$P$-value of Fisher Exact Test is reported. Codes in brackets express causes of death by International Classification of Disease-10.

Table 7. Multivariant logistic regression analysis for prediction of 2-year all-cause rehospitalization/mortality of acute heart failure patients after dismissal from the index hospitalization; stratified by the value of left ventricular ejection fraction (HFrEF and HFpEF patients).

\begin{tabular}{|c|c|c|c|}
\hline \multirow{2}{*}{ Predictor } & & \multicolumn{2}{|c|}{ LVEF < 40\% $(n=482)$} \\
\hline & & OR (95\% IS) & $P$ \\
\hline Age $(+10$ years $)$ & & $1.38(1.15 ; 1.66)$ & $<0.001$ \\
\hline Diastolic pressure $(+10 \mathrm{mmHg})$ & & $0.87(0.77 ; 1.00)$ & 0.045 \\
\hline \multicolumn{4}{|l|}{ NYHA class at admission } \\
\hline & 3 vs $1+2$ & $2.49(1.36 ; 4.56)$ & 0.003 \\
\hline eGFR (+10 mL/min) & & $0.89(0.81 ; 0.98)$ & 0.020 \\
\hline Peripheral artery disease & & $2.07(1.21 ; 3.52)$ & 0.008 \\
\hline
\end{tabular}

AUC (95\% IS): 0.702 (0.654; 0.750), $P$-value: $<0.001$

\begin{tabular}{lccr}
\hline \multicolumn{1}{c}{ Predictor } & \multicolumn{2}{c}{ LVEF $\geq 50 \%(\mathrm{n}=440)$} & \\
& & OR $(95 \%$ IS $)$ & $P$ \\
\hline NYHA class at admission & 2 vs 1 & $2.04(1.19 ; 3.52)$ & 0.010 \\
& 3 vs 1 & $4.30(1.72 ; 10.72)$ & 0.002 \\
eGFR $(+10 \mathrm{~mL} / \mathrm{min})$ & & $0.80(0.72 ; 0.88)$ & $<0.001$ \\
Diabetes mellitus & & $1.69(1.07 ; 2.68)$ & 0.024 \\
Peripheral artery disease & & $2.04(1.10 ; 3.78)$ & 0.023 \\
\hline \hline
\end{tabular}

AUC (95\% IS): 0.715 (0.663; 0.767), $P$-value: $<0.001$

For the list of all included variables see Appendix Table 2; only statistically significant predictors are shown.

AUC - Area Under Curve; eGFR - estimated glomerular filtration rate by Modification of Diet in Renal Disease study equation;

LVEF - left ventricular ejection fraction 
Table 3 presents major causes of hospitalizations. The most frequent cardiovascular cause was acute decompensation of heart failure (31.2\% of all hospitalizations), followed by arrhythmias, ACS, and cerebrovascular disease. Noncardiovascular hospitalizations were mostly due to gastrointestinal problems, infections, tumours, diabetes mellitus, bleeding events, and renal disease.

\section{Stratification of patients by LVEF: comparison of HFrEF and HFpEF groups}

Baseline characteristics, course of hospitalization, medication

Focusing on prespecified groups of patients with HFrEF and HFpEF (Table 1, right side), the patients with low LVEF were much more often men (64.5\% vs $35.7 \%)$ and on average 3 years younger ( 73.8 vs 76.9 years) than the patients with $\mathrm{LVEF} \geq 50 \%$ (both $P<0.001$ ). These findings reflect the fact that the HFrEF group consisted of patients mostly with ischemic heart disease, with prior myocardial infarction and coronary intervention. These patients also presented dominantly with acutely decompensated chronic heart failure and not with de novo heart failure $(59.3 \%$ and $40.7 \%$ in $\mathrm{HFrEF}$ vs $39.1 \%$ and $60.9 \%$ in HFpEF, $P<0.001)$. Hypertensive crisis as the cause of heart failure was identified in $10.5 \%$ of the $\mathrm{HFpEF}$ group but in only $1.2 \%$ in the HFrEF group $(P<0.001)$. Pharmacological treatment at discharge was in accordance with heart failure guidelines, with a higher prevalence of beta-blockers, spironolactone, loop diuretics, and digoxin in the HFrEF (all $P<0.001$ ).

Echocardiographic pictures were also different. Advanced grades of diastolic dysfunction with secondary mitral and tricuspid regurgitation were frequently found in HFrEF patients, while in the HFpEF group, other valvular defects were more often stated as the primary aetiology of AHF (Table 1, right side; Table 4).

\section{Outcome}

Table 5 shows clearly that we found a significant difference in the occurrence of the primary combined endpoint between the HFrEF and HFpEF groups (53.1\% vs 44.3\%, $P=0.014$ ) which was mostly driven by a higher AHF rehospitalization rate in the group with severe systolic dysfunction. All-cause hospitalizations and hospitalizations for cardiovascular causes were similar between the groups. The all-cause mortality also did not differ between groups (37.8\% vs $33.6 \%$ ). Notably, the incidence of cardiovascular deaths was significantly related to the LVEF value, reaching the highest percentage in the HFrEF group, followed by the mid-range group, and with the lowest death rate in the HFpEF group ( $82.4 \%$ vs $73.0 \%$ vs $63.5 \%$, respectively, $P<0.001)$. More patients with preserved LVEF died from a noncardiac disease, particularly tumours or respiratory system failure (Table 6).

To express differences in patient characteristics and risk profiles between the two groups in terms of 2-year mortality risk/all-cause rehospitalization, we selected more than 30 variables (demographic, laboratory, echocardiographic, and hemodynamic parameters, medica- tions and concomitant cardiovascular comorbidities recorded during the index AHF hospitalization) and calculated the odds ratios (ORs). A multivariate regression analysis revealed that only glomerular filtration rate and the presence of peripheral artery disease were independent predictors of the combined endpoint identically for HFrEF and HFpEF patients (Table 7).

\section{DISCUSSION}

Our paper evaluated the 2-year prognosis of an unselected group of consecutive patients released in stable condition from hospitalization for AHF. Our patients were well treated according to the guidelines. Somewhat misleading is the number of revascularisation procedures (percutaneous coronary intervention [PCI]/coronary artery bypass graft $[\mathrm{CABG}]$ ) that were performed in only $14.2 \%$ of patients during the index hospitalization. The reason for this finding is that many patients underwent elective coronarography/PCI/CABG shortly after discharge. This is a common practice in our region for reimbursement purposes.

In this study, we found the prognosis of heart failure patients to be very poor. Approximately one-third of all patients died, a quarter of the patients were hospitalised for AHF, and only about a third of the patients survived without any further hospitalization over 2 years. These unfavourable outcomes were much worse than those reported in recent randomised clinical trials ${ }^{6-9}$ that focused on only stable chronic heart failure patients with either systolic dysfunction (different LVEF cutoffs) or preserved LVEF $>45 \%$. These trials (median patient follow-up 23-40 months), compared with our registry, reported significantly lower mortality rates ( $15.5 \%-19.8 \%$ vs $36.1 \%$ ), fewer rehospitalizations for acute decompensation of heart failure (14.2\%-18.4\% vs $24.9 \%$ ), and fewer all-cause hospitalizations $(35.8 \%-45 \%$ vs $57 \%)$. The primary composite endpoint in these trials, comprising hospitalizations for heart failure and/or cardiovascular mortality, occurred in $20.4 \%$ to $29 \%$ of the patients ${ }^{8-10,13}$, while in our real-world registry, the incidence reached $41.3 \%$. These variances are due to different characteristics of the enrolled patients. Our registry patients were older (60-69 years vs 75.9 years), had more comorbidities, and, importantly, $48.7 \%$ were de novo heart failure patients with no previous experience with heart failure treatment. On the contrary, the clinical trials mentioned above with a long-term follow-up enrolled chronic heart failure patients with varied histories and therapies. In a meta-analysis by Pocock et al. (ref. ${ }^{5}$ ), evaluating 6 randomised studies and 24 observational registries, the overall mortality was $40.2 \%$ during the 2.5 year follow-up, which is comparable with our cohort. Our results provide more complex information in addition to overall mortality (i.e., numbers and causes of subsequent rehospitalizations and deaths). This information may help place the focus on and manage the comorbidities of heart failure patients that potentially lead to subsequent events.

One has to point out that the patients were at the highest risk of hospitalization or death during the first year 
(particularly during the first 3-6 months) after discharge. Later on, the cumulative incidence of events declined (the number of hospitalizations from any cause in the $1^{\text {st }}$ year was 1193 and in the $2^{\text {nd }}$ year 318 cases).

Recently, much has been discussed about the differences in the characteristics and outcomes of patients with reduced and preserved LVEF. Many previous heart failure trials or registries had excluded patients with preserved LVEF. Low LVEF (various cutoffs of $<35 \%$ to $<50 \%$ ) had been found to be a strong negative predictor of mortality $^{17,18}$, and patients with preserved LVEF were believed to have a better survival. Today, we perceive HFrEF and HFpEF as different entities with various patient characteristics but with very similar outcomes. Previous studies found that chronic obstructive pulmonary disease (COPD), anaemia, diabetes mellitus, and obesity were more prevalent in $\mathrm{HFpEF}$ patients than in those with HFrEF. In our study, we did not observe a different prevalence of the above-mentioned comorbidities between the patient groups but the HFpEF patients were older, more often women and with significantly less frequent ischemic heart disease. In most trials and registries, COPD was associated with a higher mortality in HFpEF subjects ${ }^{19}$. Pocock et al. identified 13 major variables associated with long-term mortality (LVEF value being one of them), but only systolic blood pressure and age had different prognostic impacts on patients with reduced $(<40 \%)$ and preserved (>40\%) LVEF. All other variables (particularly creatinine, New York Heart Association class, diabetes mellitus, etc.) demonstrated comparable negative predictive values for both groups ${ }^{5}$. Similarly in our study, we identified the decrease in glomerular filtration rate, concomitant peripheral artery disease and advanced chronic dyspnoea (NYHA class III) to be negatively associated with increased 2-year rehospitalization/mortality rates of our AHF patients. Specifically for each patient group, higher diastolic blood pressure was protective in HFrEF patients, unlike their higher age, and concomitant diabetes mellitus was significantly and independently associated with adverse outcomes in the HFpEF group. The effect of different doses of loop diuretics on the long-term mortality of the same cohort of patients was described elsewhere ${ }^{20}$.

We demonstrated that there were differences between heart failure patients with low and preserved LVEF. Consequently, there were more AHF rehospitalizations and cardiovascular deaths in the HFrEF group during the 2-year follow-up and numerically more noncardiovascular deaths and rehospitalizations in the HFpEF group. Similar differences had been described previously ${ }^{21}$. The results suggest that we should wisely consider our treatment and management plans in order to focus more on noncardiovascular comorbidities of HFpEF patients in particular ("triggers" of heart failure), possibly limit the use of loop diuretics in HFpEF patients, carefully follow all heart failure patients during initial "critical" months after discharge, and perform more revascularisation procedures during the same hospitalization in patients with HFrEF of ischemic origin. Specially designed observational studies using advanced echocardiographic parameters, stress tests, and biomarkers as well as studies performing close follow-up of the patients are needed to determine the best management of care of patients with HFpEF.

There are limitations of our study. (1) We evaluated patients from only 2 hospital centres. Nevertheless, as one site has a 24/7 operating cath lab (a district hospital) and the other site does not (regional referring hospital), and both centres enrolled all consecutive patients, we consider our patient cohort to be a representative sample of the entire population. (2) We collected data about numbers of hospitalizations, causes of hospitalizations, and deaths but not subsequent interventions, laboratory tests, echocardiographic parameters, and so forth. All characteristics and results of individual patients originated from the index hospitalization only. (3) We are aware of the fact that there are other prognostic variables identified in previous registries (hyponatremia, anaemia, novel biomarkers, etc) that were not included in our analysis. (4) In the analysis by LVEF value, some patients might have had right ventricular dysfunction causing right heart failure with preserved LVEF but with much worse outcome than other patients with preserved LVEF. Similar controversies could have been found in other AHF patients with malign arrhythmias or high-output heart failure (sepsis, anaemia). All of these patients were included in the registry.

\section{CONCLUSION}

Despite advanced therapies, the prognosis of patients released from hospitalization for heart failure remains poor. Within 2 years, half of these patients die and/or are hospitalised for acute decompensation of heart failure, and only about a third of the patients survive without any hospitalization. Unselected heart failure patients from real clinical practice have worse prognosis than those enrolled (selected) in clinical trials. Patients with reduced LVEF $(<40 \%)$, when compared with those with preserved LVEF $(\geq 50 \%)$, have different characteristics and risk profiles. $\mathrm{HFrEF}$ patients experience more rehospitalizations for heart failure and die more often of a cardiovascular cause than HFpEF patients, but overall mortality and rehospitalization rates do not differ between the groups. More detailed investigations are needed to determine an association of noncardiovascular comorbidities and mortality of HFpEF patients.

\section{ABBREVIATIONS}

ACEIs/ARBs: Angiotensin-converting-enzyme inhibitors/angiotension receptor blockers; ACS: Acute coronary syndrome; AHF: Acute heart failure; CHF, Chronic heart failure; CV: Cardiovascular; GFR: Glomerular filtration rate; $\mathrm{HFpEF}$ : Heart failure with preserved ejection fraction; HFrEF: Heart failure with reduced ejection fraction; LVEF: Left ventricular ejection fraction; NYHA: Dyspnea degree according to New York Heart Association; OR: Odds ratio.

Acknowledgement: This research was supported by the Ministry of Health of the Czech Republic-conceptual de- 
velopment of research organisations (FNBr, 65269705) and by the research project No. MUNI/A/1250/2017 of Masaryk University (Brno, Czech Republic, principal investigator Petr Lokaj). The funders had no role in study design, data collection and analysis, decision to publish, or preparation of the manuscript.

Author contributions: MM, RM, JS: manuscript writing; MM, RM, RS, KZ, MF, LP: data collection and revision; JS, PL: scientific revision and consultations; KB, LD, JJ: statistics, graphical visualisation and table design.

Conflict of interest statement: The authors state that there are no conflicts of interest regarding the publication of this article.

\section{REFERENCES}

1. Spinar J, Hradec J, Špinarová L, Vítovec J. Summary of the 2016 ESC Guidelines on the diagnosis and treatment of acute and chronic heart failure. Prepared by the Czech Society of Cardiology. Cor Vasa 2016;58:e530-e568.

2. Ponikowski P, Voors AA, Anker SD, Bueno H, Cleland JGF, Coats AJS, Falk V, González-Juanatey JR, Harjola V-P, Jankowska EA, Jessup M, Linde C, Nihoyannopoulos P, Parissis JT, Pieske B, Riley JP, Rosano GMC, Ruilope LM, Ruschitzka F, Rutten FH, van der Meer P, Authors/ Task Force Members. 2016 ESC Guidelines for the diagnosis and treatment of acute and chronic heart failure: The Task Force for the diagnosis and treatment of acute and chronic heart failure of the European Society of Cardiology (ESC) Developed with the special contribution of the Heart Failure Association (HFA) of the ESC. Eur Heart J 2016;37(27):2129-2200.

3. Heidenreich PA, Albert NM, Allen LA, Bluemke DA, Butler J, Fonarow GC, Ikonomidis JS, Khavjou O, Konstam MA, Maddox TM, Nichol G, Pham M, Piña IL, Trogdon JG. Forecasting the impact of heart failure in the united states a policy statement from the american heart as sociation. Circ Heart Fail 2013;6(3):606-19.

4. Writing Group Members, Mozaffarian D, Benjamin EJ, Go AS, Arnett DK, Blaha MJ, Cushman M, Das SR, de Ferranti S, Després J-P, Fullerton HJ, Howard VJ, Huffman MD, Isasi CR, Jiménez MC, Judd SE, Kissela BM, Lichtman JH, Lisabeth LD, Liu S, Mackey RH, Magid DJ, McGuire DK, Mohler ER, Moy CS, Muntner P, Mussolino ME, Nasir K, Neumar RW, Nichol G, Palaniappan L, Pandey DK, Reeves MJ, Rodriguez CJ, Rosamond W, Sorlie PD, Stein J, Towfighi A, Turan TN, Virani SS, Woo D, Yeh RW, Turner MB, American Heart Association Statistics Committee, Stroke Statistics Subcommittee. Executive Summary: Heart Disease and Stroke Statistics--2016 Update: A Report From the American Heart Association. Circulation 2016;133(4):447-54.

5. Pocock SJ, Ariti CA, McMurray JJV, Maggioni A, Køber L, Squire IB, Swedberg K, Dobson J, Poppe KK, Whalley GA, Doughty RN. Predicting survival in heart failure: a risk score based on 39372 patients from 30 studies. Eur Heart J 2013;34(19):1404-13.

6. Pitt B, Pfeffer MA, Assmann SF, Boineau R, Anand IS, Claggett B, Clausell N, Desai AS, Diaz R, Fleg JL, Gordeev I, Harty B, Heitner JF, Kenwood CT, Lewis EF, O'Meara E, Probstfield JL, Shaburishvili T, Shah SJ, Solomon SD, Sweitzer NK, Yang S, McKinlay SM. Spironolactone for Heart Failure with Preserved Ejection Fraction. N Engl J Med 2014;2014(370):1383-92.

7. Swedberg K, Komajda M, Böhm M, Borer JS, Ford I, Dubost-Brama A Lerebours G, Tavazzi L, SHIFT Investigators. Ivabradine and outcomes in chronic heart failure (SHIFT): a randomised placebo-controlled study. Lancet Lond Engl 2010;376(9744):875-85.

8. Zannad F, McMurray JJV, Krum H, van Veldhuisen DJ, Swedberg K, Shi H, Vincent J, Pocock SJ, Pitt B, EMPHASIS-HF Study Group. Eplerenone in patients with systolic heart failure and mild symptoms. N Engl J Med 2011;364(1):11-21.
9. McMurray JJV, Packer M, Desai AS, Gong J, Lefkowitz MP, Rizkala AR, Rouleau JL, Shi VC, Solomon SD, Swedberg K, Zile MR, PARADIGM-HF Investigators and Committees. Angiotensin-neprilysin inhibition versus enalapril in heart failure. N Engl J Med 2014;371(11):993-1004.

10. Nieminen MS, Böhm M, Cowie MR, Drexler H, Filippatos GS, Jondeau G, Hasin Y, Lopez-Sendon J, Mebazaa A, Metra M, Rhodes A, Swedberg K, Priori SG, Garcia MAA, Blanc J-J, Budaj A, Cowie MR, Dean V, Deckers J, Burgos EF, Lekakis J, Lindahl B, Mazzotta G, Morais J, Oto A, Smiseth OA, Garcia MAA, Dickstein K, Albuquerque A, Conthe P, Crespo-Leiro M, Ferrari R, Follath F, Gavazzi A, Janssens U, Komajda M, Morais J, Moreno R, Singer M, Singh S, Tendera M, Thygesen K, ESC Committe for Practice Guideline (CPG). Executive summary of the guidelines on the diagnosis and treatment of acute heart failure: the Task Force on Acute Heart Failure of the European Society of Cardiology. Eur Heart J 2005;26(4):384-416.

11. Spinar J, Jarkovsky J, Spinarova L, Mebazaa A, Gayat E, Vitovec J, Linhart A, Widimsky P, Miklik R, Zeman K, Belohlavek J, Malek F, Felsoci M, Kettner J, Ostadal P, Cihalik C, Vaclavik J, Taborsky M, Dusek L, Littnerova S, Parenica J. AHEAD score--Long-term risk classification in acute heart failure. Int J Cardiol 2016;202:21-6.

12. Methodology materials for 2013. Accessed August 23, 2018. https://www.mzcr.cz/dokumenty/metodicke-materialy-prorok-2013_7262_1058_3.html

13. Johansson I, Edner $M$, Dahlström U, Näsman $P$, Rydén $L$, Norhammar A. Is the prognosis in patients with diabetes and heart failure a matter of unsatisfactory management? An observational study from the Swedish Heart Failure Registry. Eur J Heart Fail 2014;16(4):409-18.

14. Pavlušová M, Klimes J, Špinar J, Zeman K, Jarkovský J, Benešová K, Miklik R, Pohludková L, Felšöci M, Veselá V, Blahovcová M, Dostál F, Vonka R, Pařenica J. Chronic heart failure - Impact of the condition on patients and the healthcare system in the Czech Republic: A retrospective cost-of-illness analysis. Cor Vasa 2018;60(3):e224-e233.

15. Spinar J, Parenica J, Vitovec J, Widimsky P, Linhart A, Fedorco M, Malek F, Cihalik C, Spinarová L, Miklik R, Felsoci M, Bambuch M, Dusek L, Jarkovsky J. Baseline characteristics and hospital mortality in the Acute Heart Failure Database (AHEAD) Main registry. Crit Care Lond Engl 2011;15(6):R291.

16. Parenica J, Spinar J, Vitovec J, Widimsky $P$, Linhart A, Fedorco M, Vaclavik J, Miklik R, Felsoci M, Horakova K, Cihalik C, Malek F, Spinarova L, Belohlavek J, Kettner J, Zeman K, Dušek L, Jarkovsky J, AHEAD Main investigators. Long-term survival following acute heart failure: the Acute Heart Failure Database Main registry (AHEAD Main). Eur J Intern Med 2013;24(2):151-60.

17. Rahimi K, Bennett D, Conrad N, Williams TM, Basu J, Dwight J, Woodward M, Patel A, McMurray J, MacMahon S. Risk Prediction in Patients With Heart Failure: A Systematic Review and Analysis. JACC Heart Fail 2014;2(5):440-6.

18. The survival of patients with heart failure with preserved or reduced left ventricular ejection fraction: an individual patient data metaanalysis. Eur Heart J 2012;33(14):1750-7.

19. Mentz RJ, Kelly JP, von Lueder TG, Voors AA, Lam CSP, Cowie MR, Kjeldsen K, Jankowska EA, Atar D, Butler J, Fiuzat M, Zannad F, Pitt B, O'Connor CM. Noncardiac Comorbidities in Heart Failure With Reduced Versus Preserved Ejection Fraction. J Am Coll Cardiol 2014;64(21):2281-93.

20. Pavlusova M, Miklik R, Spacek R, Benesova K, Zeman K, CohenSolal A, Mebazaa A, Littnerova S, Felsoci M, Pohludkova L, Dusek L, Špinarova L, Vitovec J, Spinar J, Jarkovsky J, Parenica J. Increased dose of diuretics correlates with severity of heart failure and renal dysfunction and does not lead to reduction of mortality and rehospitalizations due to acute decompensation of heart failure; data from AHEAD registry. Cor Vasa 2018;60(3):e215-e223.

21. Cheng RK, Cox M, Neely ML, Heidenreich PA, Bhatt DL, Eapen ZJ, Hernandez AF, Butler J, Yancy CW, Fonarow GC. Outcomes in patients with heart failure with preserved, borderline, and reduced ejection fraction in the Medicare population. Am Heart J 2014;168(5):721-30. e3. 